\title{
Consolidation and interference in the perceptual memory system
}

\author{
DOMINIC W. MASSARO ${ }^{1}$ \\ UNIVERSITY OF CALIFORNIA, SAN DIEGO
}

\begin{abstract}
Previous verbal/memory studies have indicated that perceptual processes continue to occur after the stimulus is terminated. The present study investigated whether a blank period after a nonverbal stimulus would function to strengthen or consolidate the sensory trace in memory. $A$ "delayed-comparison task" of recognition memory for pitch was employed in two experiments. The results indicated that a blank interval after the tone to be remembered increased the memory strength of that tone when the tone's duration was brief $(.2 \mathrm{sec})$. However, an empty interval after a tone with a longer duration $(.5 \mathrm{sec})$ did not affect memory performance. Memory strength also decreased over time during a retroactive interval tone. A storage-interference model described the quantitative results accurately.
\end{abstract}

An established phenomenon in visual and auditory perception is that increasing the duration of the stimulus increases the probability of perceiving the item (Mackworth, 1963). For example, Mackworth found that the number of digits $\mathrm{Ss}$ were able to report correctly from a 2 by 5 array increased with exposure time up to about $50 \mathrm{msec}$.

Not only the item-presentation time itself, but the period after its presentation can be utilized to acquire information about the stimulus (Sperling, 1960; Aaronson, 1967; Norman, 1966). In a review of the literature, Aaronson (1967) concluded that perceptual processes continue to occur after the item presentation is terminated. The studies of Sperling (1960) and Conrad (1964) indicate that this poststimulus perceptual processing is an encoding for an "auditory-information store." Hence, assuming two stages of processing, perception and auditory encoding, it seems that increasing the time between successive verbal items increases the amount of encoding of the items rather than the strengthening of the more primitive sensory trace.

On the other hand, increasing the blank period after a nonverbal (i.e., nonencodable) stimulus might only function to strengthen the primitive sensory trace since no further encoding is available to the $\mathrm{S}$. The paradigm employed in studying nonverbal memory is a "delayed-comparison task." In this task, a standard stimulus is followed after some interval by a comparison stimulus, and the $S$ 's task is to indicate whether the signals were the same or different. The interval separating the standard and comparison stimuli will be referred to as the retroactive interval.

Recent studies (Massaro, in press a; Wickelgren, 1969) have provided evidence that recognition memory for pitch seems to be a nonverbal or perceptual memory. In this paradigm, labeling or rehearsing the tone to be remembered can only function to disrupt memory performance. That is, due to the limitations of the articulatory-motor system, labeling a standard tone (such as high or low) or reinstating the tone during the retroactive interval does not aid the $S$ in determining whether or not the comparison tone is equal to the standard.

Wickelgren $(1966,1969)$ has studied the effects of the standard tone duration on memory for pitch. The results indicate that memory performance improves with increases in the standard tone duration asymptoting at about 4 to $8 \mathrm{sec}$. In these studies, a retroactive interference tone immediately followed the standard tone and probably terminated the consolidation of the standard tone. The present study investigated whether or not consolidation could continue during a blank interval between the standard and interference tones and improve recognition memory for pitch. In Experiment 1, the standard tone lasted $.5 \mathrm{sec}$ and the retroactive blank interval lasted $.5 \mathrm{sec}$. In Experiment 2, the standard tone lasted .2 sec and the retroactive blank interval lasted $.8 \mathrm{sec}$. In each experiment, the Blank condition was compared to a Continuous condition in which the interference tone immediately followed the standard tone.

The present studies are also analogous to visual backward-masking experiments (Eriksen \& Hoffman, 1963; Eriksen \& Steffy, 1964). These studies have indicated that dark retroactive visual fields enhance perception of the stimulus relative to bright retroactive visual fields. Since perception continues after the stimulus is terminated, the summation of brightnesses of successive presentations will affect the distinctiveness or contrast of the first stimulus figure. Hence, bright poststimulus fields will reduce the perception of the first stimulus relative to dark poststimulus fields.

Retroactive masking of auditory perception has been studied in Russia (Gol 'dburt, 1961a,b). The results indicated that a loud retroactive tone increased the detection threshold for tones. Furthermore, a second tone shortened the perceived duration of a first tone relative to the first tone being presented alone. The masking of perceived duration was much more effective than the masking of the detection threshold. The present study extends the scope of the Russian studies by asking whether or not a retroactive tone can also decrease the perception of pitch of a tone to be remembered.

\section{THEORY}

The present theory assumes that memory for pitch involves two processes: storage of the stimulus event and the decrease of the sensory trace over time. The parameters of the theory reflecting these processes will enable us to see how they are affected under different experimental conditions. For example, the parameters corresponding to storage will indicate how memory strength differs under the different consolidation conditions of the present experiments.

The theory presented here is a strength theory since it determines the S's response with a probability of 1 from the possible values of memory strength (Wickelgren, 1968). The measure of memory strength employed is $d^{\prime}$, the difference in trace strength between comparison (C) tones equal $(C=S)$ and unequal $(C \neq S)$ to the standard (S) tone.

Presentation of the $S$ tone increases the trace strength of that tone to some value $t$ and of a similar tone to some proportion of $t$. The amount of trace strength generalized to other tones is positively related to the similarity of the tones to the $S$ tone. For example, after the $S$ tone presentation, a tone $10 \mathrm{~Hz}$ lower than the $S$ tone will have more trace strength than a tone $20 \mathrm{~Hz}$ lower than the $S$ tone. At the presentation of the $\mathrm{C}$ tone, the $\mathrm{S}$ searches in memory for the trace of that particular $\mathrm{C}$ tone. If 
the trace strength $t$ of the $C$ tone exceeds some criterion $k$, the $S$ will report "same." That is,

$$
P(\text { same } \mid t)=P(s \mid t)=P(t>k)
$$

Assume that $t_{s}$ and $t_{c}$ are the trace strengths in memory of the equal and unequal $C$ tones, respectively. A correct recognition, $P\left(s \mid t_{s}\right)$, is defined as a $S$ responding same on a trial where $C=S$. An incorrect recognition, $P\left(s \mid t_{c}\right)$, is defined as a $S$ responding same on a trial where $C \neq S$. Since the consolidation of an unequal $C$ tone will always be less than an equal $C$ tone, $t_{s}$ will always be larger than $t_{c}$ Therefore, noise is added at the time of the decision process so that the probabilistic nature of responses can be predicted. This variability could arise from the decision process itself, from the memory processes, or from both. Noise is assumed to be a random variable that is normally distributed. Therefore, memory strength $\left(d^{\prime}\right)$ is defined as the difference between the means of the $t_{s}$ and $t_{c}$ normal distributions and will have a standard deviation of 1 .

In the present study, the unequal $C$ tone was always $20 \mathrm{~Hz}$ lower or higher than the $S$ tone. Therefore, the difference in memory strengths of the $\mathrm{C}=\mathrm{S}$ and $\mathrm{C} \neq \mathrm{S}$ tones is given by the following equation:

$$
d(S, S-x, I, n)=t_{s}-t_{c}
$$

where $d(S, S-x, I, n)$ is the predicted difference in memory strengths of an equal $\mathrm{C}$ tone of $\mathrm{S} \mathrm{Hz}$ and an unequal $\mathrm{C}$ tone of $\mathrm{S}-\mathrm{x} \mathrm{Hz}$, given an I tone duration of $\mathbf{n ~ s e c}$. The following derivations will be determined for $\mathrm{d}(\cdot)$, a measure of relative memory strength independent of $k$, the criterion value.

The memory strength stored at the $S$ tone presentation increases with the duration of the $S$ tone and the retroactive blank interval. The discrimination and sensory storage of the $S$ tone is represented by the following equation:

$$
d\left(t_{s}, t_{b}\right)=\alpha\left[1-e^{-\theta\left(t_{s}+t_{b}\right)}\right]
$$

where $d\left(t_{s}, t_{b}\right)$ is the relative memory strength after a $S$ tone of $t_{s} \sec$ and $a$ retroactive blank interval of $t_{b}$ sec. Evidence for this acquisition function of memory strength during the $S$ tone presentation has been found by Wickelgren (1969). The present experiments will test this model with respect to consolidation during both the $S$ tone duration and the retroactive blank period.

Presentation of the retroactive (interference) tone is assumed to end the consolidation of memory strength of the $S$ tone. Furthermore, an interference tone decreases the memory strength of the $S$ tone directly proportional to the amount of consolidation of the interference tone. A quantiative description of this view of forgetting is given by the following equation:

$$
\begin{aligned}
d\left(t_{s}, t_{b}, t_{l}\right) & =d\left(t_{s}, t_{b}\right) e^{-\lambda\left(1-e^{-v t_{1}}\right)} \\
& =\Delta \phi
\end{aligned}
$$

where $\Delta$, the memory strength of the $S$ tone stored, is given by Eq. 3. The value $\phi$ is the proportion of memory strength remaining after an interference tone duration of $t_{1}$ sec. Evidence for this view of forgetting is presented elsewhere (Massaro, in press b). Equation 4 indicates that $\phi$ is independent of the value of $\Delta$. Hence, it is predicted that the blank interval may increase the amount of memory strength stored, but that the proportion $\phi$ of memory strength lost due to the consolidation of an interference tone of $t_{I} \sec$ will be equal for the Blank and Continuous conditions.

The value of $\Delta$ can be thought of as an index of perceptual storage. The value $\phi$ is an index of the magnitude of the deterioration of the memory trace. These two parameters reflect the two psychological processes postulated by the present theory: perceptual storage and forgetting.

\section{General \\ Subjects. The Ss were two males and one} fernale attending the University of California. They were paid $\$ 1.93$ per hour for participation in the experiment.

Apparatus. All experimental events were controlled by a PDP-8 computer. A digitally controlled oscillator (Wavetek Model 155) was used to produce the pure tones. The stimuli were presented over matched headphones (Grason-Stadler Model PDH-39). The intensity of the tones was $64 \mathrm{~dB}$ SPL. Ss recorded their "same" or "different" responses on a portable computer card punch (Wright Model 2600).

Procedure. The onset of a standard (S) tone started a trial. In the Continuous condition, the interference (I) tone followed immediately and lasted .5, 1, 2, or 4 sec. In the Blank condition, an empty interval followed the $S$ tone. After the empty or blank interval, the I tone commenced and lasted $.5,1,2$, or $4 \mathrm{sec}$. The comparison (C) tone followed the I tone immediately in both the Blank and Continuous conditions and lasted $.5 \mathrm{sec}$.
Design. Four $\mathbf{S}$ tones $(820,840,860$, and $880 \mathrm{~Hz}$ ), four I tone durations $(.5,1$, 2 , and $4 \mathrm{sec})$, three $S-C$ differences $(+20$, 0 , and $-20 \mathrm{~Hz}$ ), and the presence or absence of a blank period after the $S$ tones were factorially combined. The 96 possible conditions were completely random in their occurrence. Four trial blocks of 80 trials each constituted a session (about $50 \mathrm{~min}$ ). There was an interval of $6 \mathrm{sec}$ between trials and an interval of about $1 \mathrm{~min}$ between trial blocks. No more than two sessions were given in 1 day.

\section{Experiment 1}

The first experiment employed an $S$ tone duration of $.5 \mathrm{sec}$ and a blank duration of $.5 \mathrm{sec}$ following the $S$ tone in the Blank condition. In the Continuous condition, the I tone immediately followed the $S$ tone. Fifty trial blocks were given in a period of about 2 weeks. The data were pooled over all trials to increase the number of observations. The number of observations contributing to each correct and incorrect recognition probability at each condition was about 250 .

\section{Experiment 2}

The second experiment employed the same Ss as the first experiment and the same experimental conditions except that, in this study, the $S$ tone lasted for only $.2 \mathrm{sec}$ and the blank interval before the $I$ tone lasted $.8 \mathrm{sec}$.

\section{RESULTS}

In each experiment, the data were pooled over all trials to increase the number of observations. Correct and incorrect recognition probabilities were computed for each $S$ at each experimental condition. The index of memory strength is $d^{\prime}$, the difference in memory strengths of comparison tones equal and unequal to the standard tone. Therefore, correct and incorrect recognition probabilities were transformed to values of $d^{\prime}$ using the tables given by Elliot (1964).

The results were analyzed with respect to the parameter values of the storage-forgetting theory. The predicted values of $\mathrm{d}^{\prime}$ were determined by estimating the parameters of Eq. 4 using a least-squares criterion between observed and predicted values of $\mathrm{d}^{\prime}$. Each experiment was analyzed separately since Experiment 2 followed Experiment 1 temporally and, thus, the experiments are not directly comparable. Therefore, for each experiment, four parameters were estimated for the model's prediction of eight experimental data points.

Figure 1 shows that in Experiment 1, an empty interval between the standard and interference tones did not significantly 


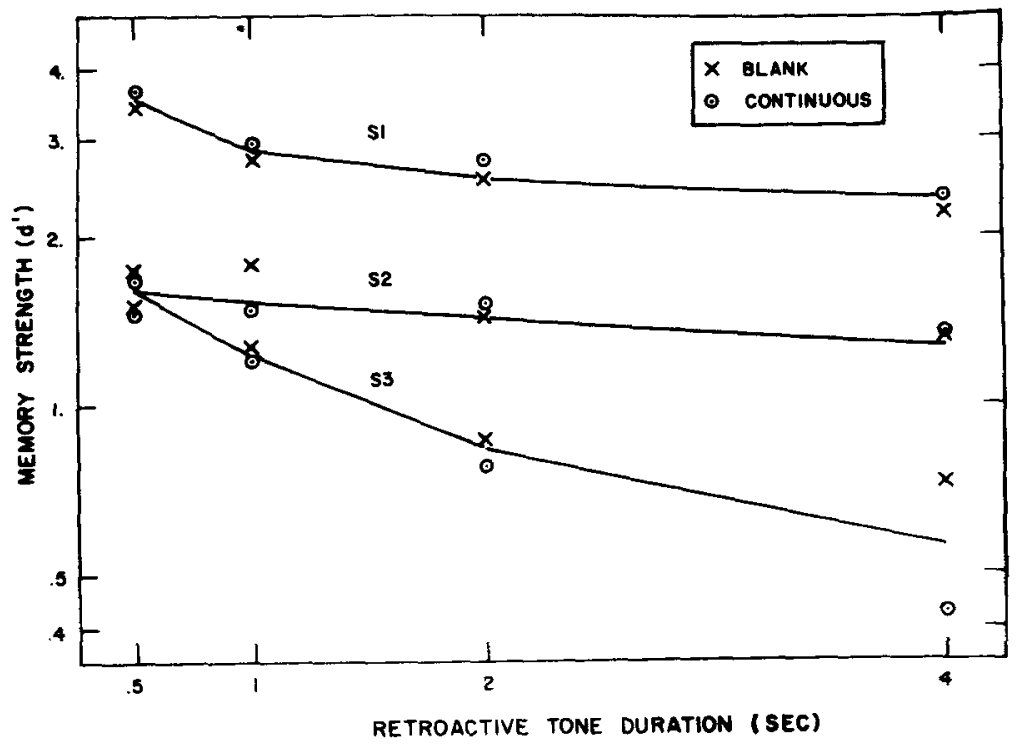

Table 1

Predicted Storage and Forgetting Values Given

by the Theory for the 8 Experimental Conditions of Experiment 1 (Using

4 Parameter Estimates

Storage Values $\Delta=\alpha\left[1-\mathrm{e}^{-\theta\left(\mathrm{t}_{\mathrm{s}}+\mathrm{tb}_{\mathrm{b}}\right)}\right]$

\begin{tabular}{lccc} 
Conditions & $\mathrm{S} 1$ & $\mathrm{~S} 2$ & $\mathrm{S3}$ \\
Blank $\left(\mathrm{t}_{\mathrm{s}}+\mathrm{t}_{\mathrm{b}}\right)$ & 4.62 & 1.78 & 2.28 \\
Continuous $\left(\mathrm{t}_{\mathrm{s}}\right)$ & 4.59 & 1.63 & 2.26 \\
\multicolumn{4}{c}{ Forgetting Values $\phi=\mathrm{e}^{-\lambda\left(1-\mathrm{e}^{\left.-\nu \mathrm{t}_{1}\right)}\right.}$}
\end{tabular}

Interference Tone

\begin{tabular}{llll} 
Duration $\left(t_{1}\right)$ & $S 1$ & $S 2$ & $S 3$ \\
.5 & .76 & .97 & .71 \\
1.0 & .64 & .94 & .54 \\
2.0 & .55 & .88 & .37 \\
4.0 & .51 & .78 & .25 \\
\hline
\end{tabular}

increase the amount of memory strength stored by the standard tone presentation. Table 1 shows that estimated parameter values give similar values of storage strength for both the Blank and
Tabie 2

Predicted Storage and Forgetting Values Given by the Theory for the 8 Experimental Conditions of Experiment 2 (Using 4 Parameter Estimates

Storage Values $\Delta=\alpha\left[1-e^{-\theta\left(t_{s}+t_{b}\right)}\right]$

\begin{tabular}{lccc} 
Conditions & $\mathrm{S} 1$ & $\mathrm{S2}$ & $\mathrm{S3}$ \\
Blank $\left(\mathrm{t}_{\mathrm{s}}+\mathrm{t}_{\mathrm{b}}\right)$ & 3.84 & 2.36 & .95 \\
Continuous $\left(\mathrm{t}_{\mathrm{s}}\right)$ & 3.04 & 1.42 & .57 \\
\multicolumn{4}{c}{ Forgetting Values $\phi=\mathrm{e}^{-\lambda}\left(1-\mathrm{e}^{\left.-\nu \mathrm{t}_{\mathrm{I}}\right)}\right]$} \\
Interference Tone & & & \\
Duration ( & & $\mathrm{S})$ \\
.5 & $\mathrm{~S} 1$ & $\mathrm{~S} 2$ & $\mathrm{S3}$ \\
1.0 & .93 & .96 & .94 \\
2.0 & .88 & .93 & .90 \\
4.0 & .82 & .88 & .84 \\
\hline
\end{tabular}

\section{Continuous conditions.}

Furthermore, the blank interval did not contribute to forgetting. Figure 1 shows that the points under the Blank and Continuous are not significantly different

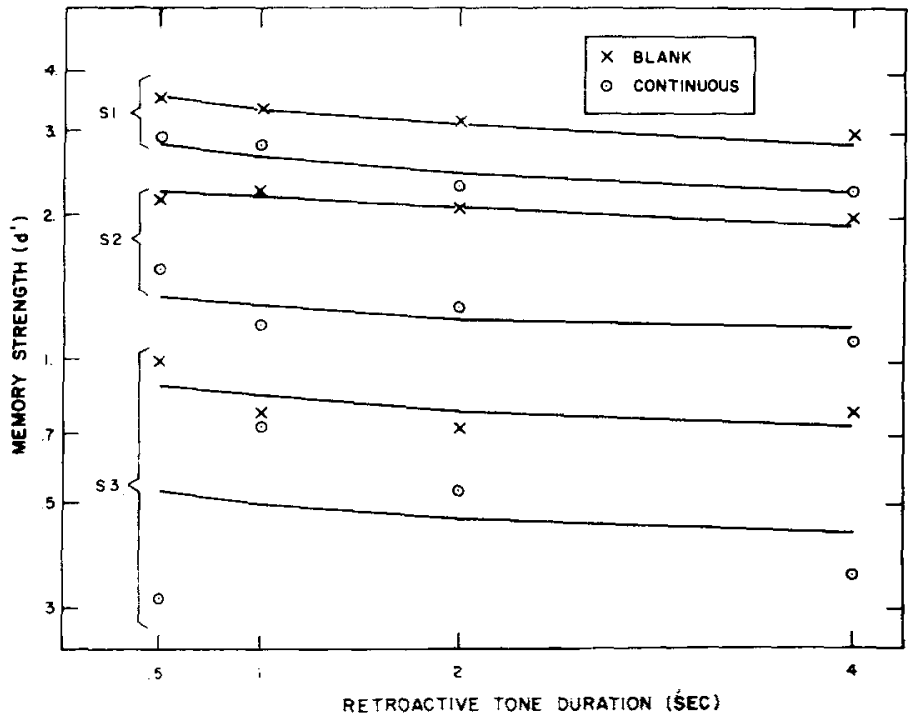

Fig. 1. Memory strength values for individual $S s$ as a function of Blank and Continuous conditions and the retroactive tone durations for Experiment 1. The continuous lines are the predictions given by the storage-interference theory.

at each interference tone duration. Figure 1 and Table 1 also indicate that the interference tone decreased the memory strength of the standard tone. Figure 1 shows that the decrease of memory strength over the duration of the interference tone is described nicely by the present theory.

Figure 2 indicates that in Experiment 2, the empty interval had a substantial facilitation effect on memory for pitch. For all three $\mathrm{Ss}$, memory performance was significantly better for the Blank condition than for the Continuous condition at each interference tone duration. Table 2 shows that the storage values are larger for the Blank condition than for the Continuous condition for all three Ss.

Figure 2 also shows that the rate of forgetting during the interference tone is the same for both the Blank and Continuous conditions. This result is in agreement with the prediction made earlier that the proportion of memory strength lost due to an interference should be independent of the initial memory strength stored.

\section{DISCUSSION}

These two experiments indicate that, with a sufficient standard tone duration, an empty interval following the standard tone does not affect memory performance relative to a continuous interference tone interval. However, at shorter standard tone durations, a blank interval after the standard tone can facilitate memory performance (increase memory strength). These studies are consistent with the studies of Gol 'dburt (1961a, b). In those studies, the masking of perceived duration was especially effective at short durations (5-100 msec) of the first tone. At longer durations ( $400 \mathrm{msec}$ ) of the first tone, a retroactive tone had little or no effect on the perceived shortening of the first tone.

The present results were described by a theory that assumes that memory strength consolidates during both the standard tone presentation and blank periods afterwards.

Fig. 2. Memory strength values for individual Ss as a function of Blank and Continuous conditions and the retroactive tone duration for Experiment 2. The continuous lines are the predictions given by the storage-interference theory. 
The consolidation over time decreases as described by a bounded acquisition function of memory strength. Hence, for shorter standard tone durations, when memory strength had not reached asymptote, memory strength continued to increase during a retroactive blank interval. On the other hand, memory performance with standard tone durations that gave an asymptotic value of memory strength was not affected by retroactive blank intervals.

The results also indicated that memory strength decreased over time during the interference tone. The theory described these results by analyzing the decreases in memory strength in terms of the consolidation or processing of the interference tone. Evidence for this view of forgetting has been substantiated in other experiments (Massaro, in press, a, b). These studies have investigated the effects of different interference stimuli and the number of interfering stimuli in a given retroactive interval. The results have indicated that forgetting is directly related to the consolidation or processing of the interference stimuli (Massaro, in press, $a, b)$. In summary, the concept of consolidation has been helpful in describing both the acquisition of perceptual memory strength and its subsequent deterioration.

\section{REFERENCES}

AARONSON, D. Temporal factors in perception and short-term memory. Psychological Bulletin, 1967, 67, 130-144.

CONRAD, $R$. Acoustic confusions in immediate memory. British Joumal of Psychology, 1964, 55, 75-84.

ELLIOTT, P. B. Tables of $d^{\prime}$. In J. A. Swets (Ed.), Signal detection and recognition by human observers. New York: Wiley, 1964. Pp. 651-684.

ERIKSEN, C. W., \& HOFFMAN, M. Form recognition at brief durations as a function of adapting field and interval between stimulations. Journal of Experimental Psychology, 1963, 66, 485-499.

ERIKSEN, C. W., \& STEFFY, R. A. Short-term memory and retroactive interference in visual perception. Journal of Experimental Psychology, 1964, 68, 423-434.

GOL 'DBURT, $S$. $N$. Changes in the strength-duration curve of tonal stimuli on masking with pure tones. Biofizika, 1961a, 6, 198-206. English translation: Biophysics, 1961a, 6, 217-227.

GOL 'DBURT, S. N. Investigation of the stability of auditory processes in micro-intervals of time (new findings on back masking). Biofizika, 1961b, 6, 717-724. English translation: Biophysics, 1961b, 6, 809-817.

MACKWORTH, J. F. The duration of the visual image. Canadian Journal of Psychology, 1963 17, 62-81.

MASSARO, D. W. Retroactive interference in short-term recognition memory for pitch. Journal of Experimental Psychology, in press, a.

MASSARO, D. W. Forgetting: Interference or decay. Journal of Experimental Psychology, in press, b.

NORMAN, D. A. Acquisition and retention in short-term memory. Journal of Experimental Psychology, 1966, 72, 369-381.

SPERLING, G. The information available in bnel visual presentations. Psychological Monograph, $1960,74,11$.

WICKELGREN, W. A. Consolidation and interference in short-term recognition memory for pitch. Journal of Experimental Psychology, $1966,72,250-259$.

WICKELGREN, W. A. Unidimensional strength theory and component analysis of noise in absolute and comparative judgments. Journal of Mathematical Psychology, 1968, 5, 102-122.

WICKELGREN, W. A. Associative strength theory of recognition memory for pitch Journal of Mathematical Psychology, 1969, 6, 13-61.

\section{NOTES}

1. This investigation was supported (in part) by a National Institutes of Health Fellowship (5F02 MH 39369-02) from the Public Health Service.

2. Address: Department of Psychology, University of California, San Diego, La Jolla, California 92037

Accepted for publication May 5, 1969.) 